\title{
The effect of caveolin-1 knockdown on interleukin-1 $\beta$-induced chemokine (C-C motif) ligand 2 expression in synovial fluid-derived fibroblast-like synoviocytes from patients with rheumatoid arthritis
}

\author{
Dorota Trzybulska, ${ }^{1, A-F}$, Anna Olewicz-Gawlik' ${ }^{2, A, C-F}$, Jan Sikora ${ }^{3, C-F}$, Magdalena Frydrychowicz ${ }^{3, B-F}$, \\ Agata Kolecka-Bednarczyk ${ }^{3, A, C, E, F}$, Mariusz Kaczmarek ${ }^{3, C, E, F}$, Paweł Hrycaj ${ }^{3,4, A, C-F}$ \\ ${ }^{1}$ Department of Rheumatology and Clinical Immunology, Poznan University of Medical Sciences, Poland \\ ${ }^{2}$ Department of Infectious Diseases, Poznan University of Medical Sciences, Poland \\ ${ }^{3}$ Department of Immunology, Poznan University of Medical Sciences, Poland \\ ${ }^{4}$ Department of Rheumatology, Municipal Hospital, Kościan, Poland \\ A - research concept and design; $B$ - collection and/or assembly of data; $C$ - data analysis and interpretation; \\ $\mathrm{D}$ - writing the article; $\mathrm{E}$ - critical revision of the article; $\mathrm{F}$ - final approval of the article
}

Address for correspondence

Dorota Trzybulska

E-mail: trzybulskadorota@gmail.com

Funding sources

None declared

Conflict of interest

None declared

Received on January 25,2017

Reviewed on June 18,2017

Accepted on June 30, 2017

\section{Abstract}

Background. Rheumatoid arthritis (RA) is a chronic autoimmune disease leading to destructive changes in peripheral joints and their irreversible deformity. The influx of chemoattractant-mediated inflammatory cells to the joints is one of the main features of RA.

Objectives. The aim of this study was to investigate the effect of a knockdown of caveolin-1 (CAV1), a known regulator of multiple cell signaling pathways, on chemokine (C-C motif) ligand 2/monocyte chemoattractant protein-1 (CCL2/MCP-1) expression in synovial fluid-derived fibroblast-like synoviocytes (sfd-FLSs) obtained from patients with RA.

Material and methods. Primary cell cultures of sfd-FLSs were established from RA synovial fluids. Cells were transiently transfected with small interfering RNA (siRNA) specific for CAV1, and then incubated with interleukin (IL)-1 13 to induce CCL2 expression. The expression levels of CAV1 and CCL2 were assessed at transcript level, using quantitative polymerase chain reaction (qPCR) and at protein level by enzyme-linked immunosorbent assay (ELISA) and western blotting analysis.

Results. A transient CAV1 knockdown in sfd-FLSs resulted in a decrease in the IL-1B-induced CCL2 mRNA expression level vs non-transfected cells and cells transfected with non-targeting siRNA. The concentration of secreted CCL2 was not affected significantly.

Conclusions. Our study demonstrates that CCL2 expression in sfd-FLSs is CAV1-dependent, but only at transcript level. As the function of CAV7 has not been unequivocally determined, more studies are needed to confirm the role of CAV1 in inflammatory processes related to RA.

Key words: rheumatoid arthritis, caveolin-1, C-C chemokine ligand 2/monocyte chemoattractant protein-1, synoviocytes

DOI

10.17219/acem/75611

Copyright

Copyright by Author(s)

This is an article distributed under the terms of the

Creative Commons Attribution Non-Commercial License

(http://creativecommons.org/licenses/by-nc-nd/4.0/) 


\section{Introduction}

Rheumatoid arthritis (RA) is a chronic autoimmune disease resulting in destructive changes in peripheral joints, and subsequently in their irreversible deformity. Rheumatoid arthritis pathogenesis is extremely complex and, despite the identification of many compelling contributory factors, it has not been fully explained. ${ }^{1}$

In RA, cells constituting the intimal lining layer of the synovium proliferate in an uncontrolled manner and form pannus tissue. ${ }^{2}$ There are 2 predominant cell types participating in synovial hyperplasia. Type A synoviocytes are terminally differentiated cells with little capacity to proliferate and uneven distribution in the synovial membrane; they activate synoviocytes type $B$, also called fibroblast-like synoviocytes (FLSs), by producing pro-inflammatory cytokines, chemokines and growth factors. ${ }^{2,3}$ Fibroblast-like synoviocytes represent a specialized type of cells and their implication in RA pathogenesis is considered in terms of 2 aspects: destructive changes in joints and chronic inflammation. ${ }^{2-6}$ Fibroblast-like synoviocytes present in pannus tissue secrete matrix metalloproteinases, cathepsins and aggrecanases, destroying cartilage which, in consequence, leads to bone resorption. ${ }^{6}$ Fibroblast-like synoviocytes promote the influx of chemoattractant-mediated inflammatory cells into the joint cavity in response to the joint milieu, factors such as interleukin (IL)- $1 \beta$ or tumor necrosis factor alpha (TNF $\alpha$ ), which is one of the main features of RA., ${ }^{4-8}$ Secreted C-C chemokine ligand 2 (CCL2) chiefly induces monocytes migrating into the joints, where they differentiate into exudate macrophages, but it can also affect $\mathrm{T}$ cells, natural killer cells and basophils. ${ }^{5}$ Activated monocytes/ macrophages play an important role in the maintenance of inflammation in RA by producing pro-inflammatory cytokines and mediators responsible for the development of synovitis. Therefore, the inhibition of their multi-step migration, driven by a chemoattractant gradient toward the sites of inflammation, is of therapeutic value., ${ }^{2,9}$

Together, the processes in joints perpetuate RA development by recruiting and retaining inflammatory cells, pannus hyperplasia and bone damage. It is believed that effective therapy to restore the balance between pro- and anti-inflammatory cytokines should aim not only at individual cytokines but also at signaling molecules responsible for their production. ${ }^{10}$ Thus, the key issue is to find a crucial molecular process which would enable us to control the course of RA. Caveolin-1 (CAV1), expressed in 2 isoforms ( $\alpha$ and $\beta$ ), is one of the major structural components of caveolae and has a number of signaling functions. ${ }^{11,12}$ This protein contains a scaffolding domain in the position 82-101, which, by binding to signaling molecules (e.g., Src kinases, endothelial nitric oxide synthase $3, \mathrm{G}$ protein $\alpha$ subunits, or protein kinase $C$ ), is able to negatively regulate cell signaling. ${ }^{11,13-15}$ On the other hand, CAV $1 \alpha$ has the ability to positively regulate signal transduction intracellularly through phosphorylation on tyrosine-14 (Tyr14), which can happen in response to cellular stress, growth factors or stimulation with hormones. ${ }^{16,17}$

Regarding inflammatory disorders, CAV1 could either prevent or induce inflammation, depending on cellular context. ${ }^{11,18-21}$ In this study, we decided to investigate the impact of CAV1 on CCL2 expression in synovial fluidderived fibroblast-like synoviocytes (sfd-FLSs) obtained from patients with RA.

\section{Material and methods}

\section{Patients}

The protocol for this study was approved by the Bioethics Committee of Poznan University of Medical Sciences (Poland). Synovial fluids were aspirated for therapeutic reasons from inflammatory knee joint effusions from 3 RA patients ( 2 women/1 man; age: $48-52$ years, disease duration: $1.5-20.5$ years) fulfilling both the 1987 American College of Rheumatology (ACR) 22 and the 2010 American College of Rheumatology/European League Against Rheumatism (ACR/EULAR) classification criteria. ${ }^{23}$ Written informed consent was obtained from every patient before any study procedure was carried out.

\section{Cell culture}

Primary cell cultures of sfd-FLSs, which could be an alternative to FLSs derived from tissues after surgery, ${ }^{24}$ were established from RA synovial fluids based on the procedure described by Scanu et al. ${ }^{25}$ To summarize briefly, fluids were collected in tubes containing ethylenediaminetetraacetic acid (EDTA), diluted twice with sterile phosphate-buffered saline (PBS) and centrifuged at $1500 \mathrm{rpm}$ for $10 \mathrm{~min}$ at room temperature. Pellets were suspended in a complete Dulbecco's Modified Eagle's medium (DMEM) (Sigma, St. Louis, USA) with 10\% fetal bovine serum (Biochrom AG, Berlin, Germany) and antibiotic-antimycotic containing 10000 units/mL of penicillin G, $10 \mathrm{mg} / \mathrm{mL}$ of streptomycin sulfate and $25 \mu \mathrm{g} / \mathrm{mL}$ of amphotericin B (ABAM; Sigma). The cells were placed into T-25 flasks and incubated under standard conditions $\left(37^{\circ} \mathrm{C}, 5 \% \mathrm{CO}_{2}\right.$ in a humidified atmosphere). After $24 \mathrm{~h}$, non-adherent cells were washed out and fresh DMEM was added to the cells. The medium was changed twice a week. Cells from passages 3-10 were used for the study. Synovial fluid-derived fibroblast-like synoviocytes were checked for the presence of macrophages by flow cytometry. The cells were stained with the following antibodies: phycoerythrinconjugated CD14 (clone M $\phi$ P9, 345785; BD, Franklin Lakes, USA), allophycocyanin/Cy7-conjugated CD45 (clone 2D-1, 348815; BD) and allophycocyanin-conjugated CD163 (clone 215927, FAB1607A; R\&D Systems, Minneapolis, USA) for $20 \mathrm{~min}$. Next, $500 \mu \mathrm{L}$ of fluorescence-activated cell sorting (FACS)-lysing solution (BD) was added in a proportion 
of 1:10 and incubated for $10 \mathrm{~min}$, protected from light and at room temperature. The cells were washed twice with PBS by centrifugation at $1200 \mathrm{~g}$ for $4 \mathrm{~min}$, suspended in $500 \mu \mathrm{L}$ of PBS, and then subjected to flow cytometry on FACSCanto II (BD). A total of 30,000 events per sample were acquired. The results were analyzed with FACSDiva software (BD Biosciences, Franklin Lakes, USA). Cultured cells did not show markers characteristic for macrophages.

\section{A dose-response study of IL-1 $1 \beta$ on CCL2 expression in sfd-FLSs}

As FLSs lose their primary phenotype with increasing passage number and become quiescent by passage 3 , they require exposure to IL-1 $\beta$ to restore their ability to produce chemokines. ${ }^{2}$ To determine the most effective IL-1 $\beta$ dose inducing CCL2 expression, FLSs were seeded on 6-well plates $\left(1 \times 10^{5}\right.$ cells/well $)$ and serum starved for $24 \mathrm{~h}$. After that, the cells were washed with PBS and treated with different doses of IL-1 $\beta$ (Sigma) $(0.1 \mathrm{ng} / \mathrm{mL}, 1 \mathrm{ng} / \mathrm{mL}$, $5 \mathrm{ng} / \mathrm{mL}$, and $10 \mathrm{ng} / \mathrm{mL}$ ) diluted in serum-free Opti-Minimal Essential Medium (MEM) (Gibco; Life Technologies, Carlsbad, USA) or left untreated. The conditioned media were collected and frozen immediately after a 2-hour incubation. The cells were washed with PBS, lysed with TRIzol (Sigma) and stored at $-70^{\circ} \mathrm{C}$ for further RNA extraction.

\section{Optimization of transfection conditions}

Transfection conditions were optimized for $1 \times 10^{5}$ cells/ well with $0.2 \%$ and $0.4 \% \mathrm{v} / \mathrm{v}$ concentrations of Lipofectamine2000 (Life Technologies) by using BLOCK-iT ${ }^{\mathrm{TM}}$ Fluorescent Oligo (Life Technologies, Carlsbad, USA) and Opti-MEM. Small interfering RNA (siRNA) uptake was assessed under a fluorescent microscope. Intensive fluorescence was visible at a minimal final concentration of labeled siRNA (10 nM) and 0.2\% v/v Lipofectamine2000, which indicated high transfection efficiency (not shown). Such conditions were tested for CAV1-siRNA and control siRNA at final concentrations of $10 \mathrm{nM}, 50 \mathrm{nM}$ and $100 \mathrm{nM}$. All the transfections were performed according to the protocols provided by the manufacturer. Cell viability was assesses by the trypan blue exclusion test (Sigma).

\section{Experiment protocol}

The cells were seeded on 6 -well plates $\left(1 \times 10^{5}\right.$ cells/well $)$ in antibiotic- and serum-free DMEM $24 \mathrm{~h}$ before transfection. Next, they were washed with Opti-MEM and transiently transfected with siRNA specific for $C A V 1$ or control siRNA (at a final concentration of $50 \mathrm{nM})(\mathrm{sc}-29241$ and sc-37007; Santa Cruz Biotechnology, Dallas, USA) in $2.5 \mathrm{~mL}$ of Opti-MEM by using $0.2 \% \mathrm{v} / \mathrm{v}$ Lipofecta-mine2000, or left untreated. Additionally, to check transfection efficiency, each time the cells were transfected with fluorescein labeled siRNAs BLOCK-iT ${ }^{\mathrm{TM}}$ and the uptake of siRNA was assessed under a fluorescent microscope. Based on information from the previous report and preliminary stages of this study, the cells were re-transfected after $48 \mathrm{~h}$ to counteract excessive $C A V 1$ expression under the influence of IL-1 $\beta$ activity. ${ }^{26}$ Next, they were stimulated for $2 \mathrm{~h}$ with $1 \mathrm{ng} / \mathrm{mL}$ IL-1 $\beta$ diluted in Opti-MEM to induce CCL2 expression. Simultaneously, control incubations with non-transfected cells and without IL-1 $\beta$ induction were conducted. After incubation, supernatants were collected and frozen immediately at $-70^{\circ} \mathrm{C}$. The cells were washed with cold PBS and lysed using TRIzol for RNA extraction or an radioimmunoprecipitation assay (RIPA) buffer (Sigma) for protein isolation. Three independent experiments were conducted on cells obtained from 3 RA synovial fluids.

\section{Total RNA extraction and CDNA synthesis}

Total cellular RNA extraction was performed using TRIzol. The concentration and purity of RNA were measured using a NanoDrop1000 spectrophotometer (Thermo Scientific, Waltham, USA). Total RNA in a dose of $0.5 \mu \mathrm{g}$ was reverse-transcribed into complementary DNA (cDNA) using a QuantiTect ${ }^{\circledR}$ Reverse Transcription Kit (Qiagen, Hilden, Germany) in accordance with the protocol provided by the manufacturer.

\section{Quantitative real-time polymerase chain reaction}

The primers used in this study (Table 1) were synthesized by DNA Sequencing and Oligonucleotides Synthesis Laboratory (Institute of Biochemistry and Biophysics, the Polish Academy of Sciences, Warszawa, Poland; www.oligo.pl). Quantitative polymerase chain reactions

Table 1. Primers used for real-time PCR

\begin{tabular}{|l|c|c|}
\multicolumn{1}{|c|}{ Gene name (symbol) } & Accession No. (NCBI) & \multicolumn{1}{c|}{$\begin{array}{c}\text { Sequences of primers } 5^{\prime} \rightarrow 3^{\prime} \\
\text { (exon numbers) }\end{array}$} \\
\hline $\begin{array}{l}\text { Porphobilinogen deaminase } \\
(\text { PBGD) }\end{array}$ & NM_000190 & $\begin{array}{c}\text { GCCAAGGACCAGGACATC (11) } \\
\text { TCAGGTACAGTTGCCATC (12/13) }\end{array}$ \\
$\begin{array}{l}\text { Chemokine (C-C motif) ligand 2 } \\
(C C L 2)\end{array}$ & NM_002982 & AGAAGAATCACCAGCAGCAAGT (2) \\
Gaveolin-1 (CAV1) & NM_001753 & GACCCTAAACACCTCAAC (2) \\
\hline
\end{tabular}

PCR - polymerase chain reaction; NCBI - National Center for Biotechnology. 
(qPCRs) were carried out on a Corbett Rotor-Gene 6000 with Rotor-Gene 6000 Series Software 1.7 (Corbett Life Science, Sydney, Australia), employing a QuantiFast SYBR ${ }^{\circledR}$ Green PCR Kit (Qiagen). To determine the relative mRNA levels of the genes studied, standard curves were generated using a mix of randomly pooled cDNA samples in six 0.6 -fold dilution series of cDNA. The thermal cycling conditionswere as follows: denaturation at $95^{\circ} \mathrm{C}$ for $5 \mathrm{~min}$, followed by 45 cycles of $95^{\circ} \mathrm{C}$ for $5 \mathrm{~s}$ and $60^{\circ} \mathrm{C}$ for $30 \mathrm{~s}$ and melting curve creation: $65-97^{\circ} \mathrm{C}$. The levels of $C C L 2$ and $C A V 1$ mRNA were normalized to porphobilinogen deaminase $(P B G D)$ mRNA levels. The results are expressed in arbitrary units as a ratio of the studied gene level to reference gene level. The reactions were done in triplicate.

\section{Sodium dodecyl sulfate- polyacrylamide gel electrophoresis and western blotting analysis}

Equal amounts of protein $(20 \mu \mathrm{g})$ were boiled for $10 \mathrm{~min}$ in Laemmli buffer, and then separated in $12 \%$ sodium dodecyl sulfate (SDS) polyacrylamide gel. Proteins from gel were transferred onto a polyvinylidene difluoride (PVDF) membrane using the Mini Semi-dry Blotting System (EPS-BIO, Chennai, India). The blots were cut into 2 pieces so as to identify CAV1 $(\sim 22 \mathrm{kDa})$ and $\beta$-actin $(\sim 43 \mathrm{kDa})$, and blocked with $5 \% \mathrm{w} / \mathrm{v}$ skimmed milk dissolved in Trisbuffered saline with Tween 20 (TBS-T). Pieces with lower-molecular-weight proteins were probed with a primary antibody for CAV1 (AP16383PU-N; Acris Antibodies, Herford, Germany) diluted 1:5,000 in 2\% skimmed milk in TBS-T for $2 \mathrm{~h}$. Afterwards, this was washed with TBS-T and incubated for $1 \mathrm{~h}$ with a horseradish peroxidase (HRP)-conjugated secondary donkey anti-goat antibody (sc-2033; Santa Cruz Biotechnology) diluted 1:20,000 in 2\% skimmed milk in TBS-T. The second piece of membrane was incubated with a HRP-conjugated polyclonal anti- $\beta$-actin antibody (sc-1616; Santa Cruz Biotechnology). The proteins were visualized as bands using the SuperSignal West Femto Maximum Sensitivity Substrate (Thermo Scientific, Rockford, USA) by exposing the membrane to X-ray film. The proteins fold change was quantified using ImageJ software v. 1.47 (NIH, Bethesda, USA). The band intensities for CAV1 were normalized to $\beta$-actin as a loading control. The CAV/ $\beta$-actin ratio for the control siRNA was assumed to be 1 .

\section{Enzyme-linked immunosorbent assay}

Concentrations of secreted CCL2 were determined using a commercially available enzyme-linked immunosorbent assay (ELISA) kit for recombinant human CCL2/monocyte chemoattractant protein-1 (MCP-1) (R\&D) in accordance with the manufacturer's protocol. The absorbance was measured with an ELISA plate reader ELx800 (Bio-Tek Instruments, Inc., Winooski, USA) using KC Junior analysis software (Bio-Tek Instruments, Inc.). The mean minimum detectable dose of CCL2 was $1.7 \mathrm{pg} / \mathrm{mL}$.

\section{Statistical analysis}

The calculations were carried out with Microsoft Excel 2010 and GraphPad Prism v. 6.0 (GraphPad Software, La Jolla, USA). The data was analyzed by a two-way analysis of variance (ANOVA) followed by Šidák's test, a oneway ANOVA followed by Tukey's honest significant difference (HSD) test or, when compared to a single control, by Dunnett's test. The results are expressed as means \pm standard deviation (SD). The differences were considered to be statistically significant at $\mathrm{p}<0.05$.

\section{Results}

\section{IL-1 $\beta$ induces CCL2 expression in sfd-FLSs, but not in a concentration- -dependent manner}

A statistically significant increase in CCL2 expression both at transcript and protein level was seen after a $2-\mathrm{h}$ incubation. Interleukin-1 $\beta$ at a concentration of $1 \mathrm{ng} / \mathrm{mL}$ was used for further incubations. The data revealed a nonlinear relationship between an increasing IL-1 $\beta$ concentration and CCL2 expression in sfd-FLSs (Fig. 1).
A

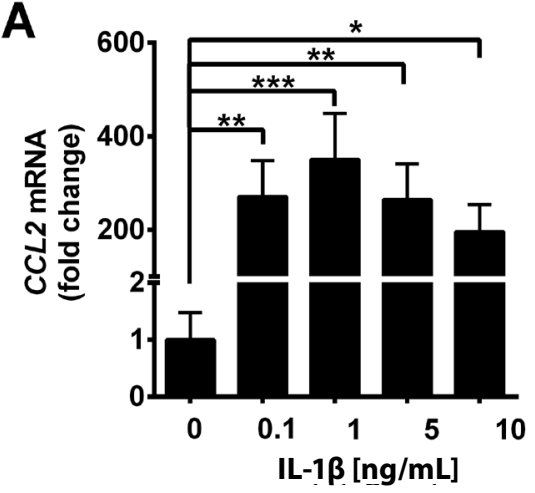

B

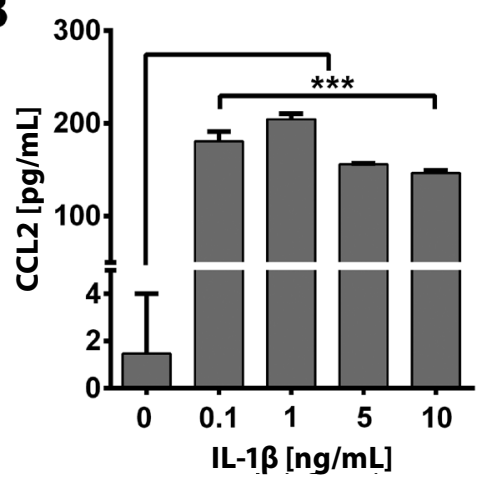

Fig. 1. The effect of IL-1 $\beta$ on the expression and production of CCL2 in sfd-FLSs

Twenty-four hours before the experiment $1 \times 10^{5}$ cells/ well were seeded on 6-well plates in DMEM without serum. The next day, the cells were incubated for $2 \mathrm{~h}$ with indicated concentrations of IL-1 $\beta$. The relative CCL2 transcript levels were measured using $\mathrm{PPCR}$ with PBGD as an internal reference gene. A) The CCL2 concentrations in conditioned medium were measured using ELISA. B) The graph bars show data as means \pm SD from 3 individual experiments; the differences between the means were analyzed by the one-way ANOVA followed by Dunnett's test; * $p<0.05 ; * * 0.01$; $* * * p<0.001$. 


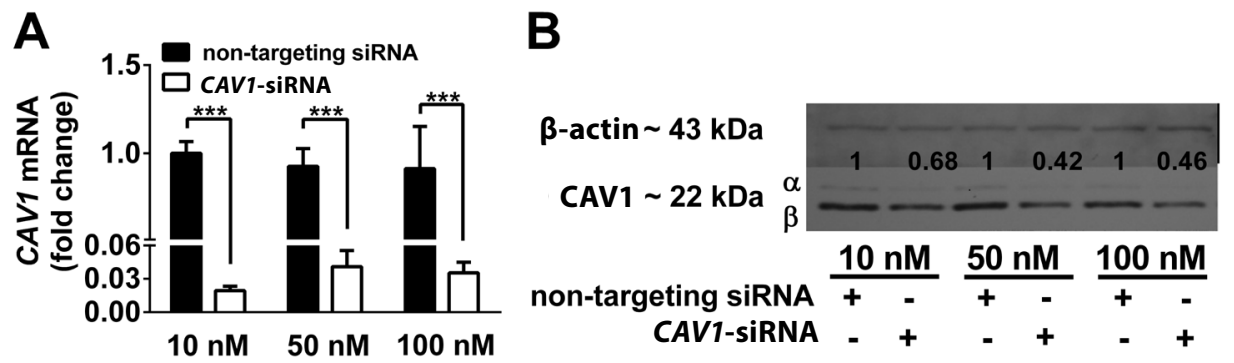

Fig. 2. Small interfering RNA (siRNA)-mediated knockdown of CAV1 expression in sfd-FLSs

Synovial fluid-derived fibroblast-like synoviocytes were transfected with CAV1-siRNA and control siRNA with indicated final concentrations. A - the relative CAV1 transcript level was assessed by qPCR with PBGD as an internal reference gene. The results are presented as a fold decrease of CAV1 expression compared with non-targeting siRNA at $48 \mathrm{~h}$ after transfection. The graph bars show data as means \pm SD from 3 individual experiments; the differences between the means were analyzed by the two-way ANOVA followed by Šídák's test: *** $\mathrm{p}<0.001$. B - The CAV1 knockdown was assessed by western blotting analysis (both isoforms were visible after longer exposure to X-ray film). Cell lysates were prepared as described in the "Material and methods" section. Equal amounts of proteins were subjected to SDS-PAGE, blotted into a PVDF membrane and incubated with anti-CAV1 and anti- $\beta$-actin antibodies. The band intensities for CAV1 were normalized to $\beta$-actin as a loading control. The CAV1/ $\beta$-actin ratio for the control siRNA was assumed to be 1 . CAV1 - caveolin-1; sfd-FLSs - synovial fluid-derived fibroblast-like synoviocytes; qPCR - quantitative polymerase chain reaction; PBGD - porphobilinogen deaminase; SDS-PAGE - sodium dodecyl sulfate-polyacrylamide gel electrophoresis; PVDF - polyvinylidene difluoride.

A

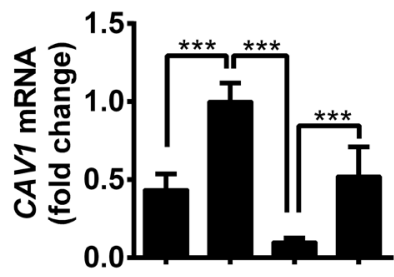

CAV1-siRNA - -+-

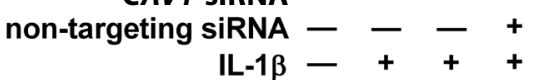

C

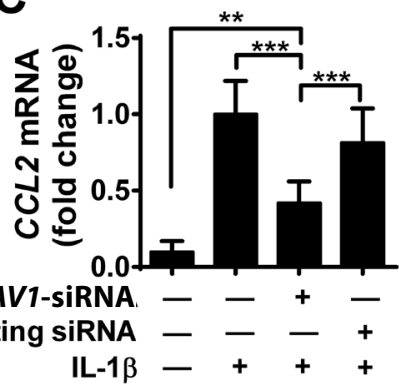

B

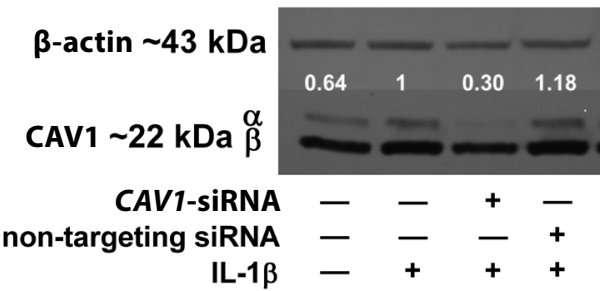

D

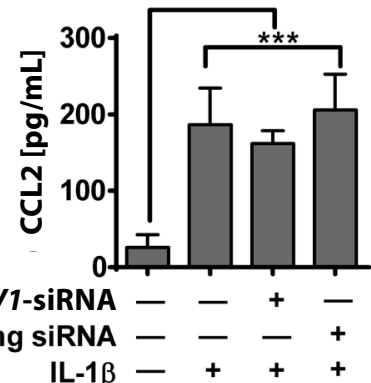

Fig. 3. CAV1 knockdown efficacy and effect on CCL2 expression in sfd-FLSs

Twenty-four hour before the experiment, $1 \times 10^{5}$ cells/well were seeded on 6-well plates in DMEM without serum. The following day, the cells were transfected with CAV1-siRNA, control siRNA or non-transfected. After $48 \mathrm{~h}$, the cells were re-transfected and incubated with IL-1 $\beta$ to induce CCL2 expression. The CCL2 and CAV1 transcript levels were measured using qPCR with PBGD as an internal reference gene ( $\mathrm{A}$ and $\mathrm{C}$ ). The CAV1 knockdown was assessed by western blotting analysis (both isoforms were visible after longer exposure with predominant $\beta$ isoform). The band intensities for CAV1 were normalized to $\beta$-actin as a loading control. The CAV1/ $\beta$-actin ratio for the control siRNA was assumed to be 1 (B). The concentrations of CCL2 in conditioned media were measured using ELISA (D). The graph bars show data as means \pm SD from 3 independent experiments conducted on sfd-FLSs obtained from each patient; the differences were tested by the one-way ANOVA followed by Tukey's HSD test; ${ }^{* *} p>0.01 ;{ }^{* * *} p<0.001$.

CAV1 - caveolin-1; CCL2 - chemokine (C-C motif) ligand 2; sfd-FLSs - synovial fluid-derived fibroblast-like synoviocytes; DMEM - Dulbecco's Modified Eagle's Medium; IL- $\beta$ - interleukin-1 $\beta$; qPCR - quantitative polymerase chain reaction; PBGD - porphobilinogen deaminase; ELISA - enzyme-linked immunosorbent assay.

\section{CAV1 knockdown in sfd-FLSs impairs CCL2 transcription}

Transfection with siRNA did not affect sfd-FLSs viability. As CAV1 is a long-lived protein, transcript level did not correspond with protein level after transfections. ${ }^{27}$ For further transfections, $50 \mathrm{nM}$ siRNA was chosen (Fig. 2). A longer exposure (for $1 \mathrm{~min}$ ) of the PVDF membrane blot to X-ray film revealed both $C A V 1$ isoforms with predominant $\beta$ isoform.

The CAV1 level was transiently knocked down by about $70 \%$ in comparison with cells transfected with control 
siRNA or non-transfected cells. Incubation with IL-1 $\beta$ caused an increase in CAV1 expression, which is in line with the previous report (Fig. 3A,3B). ${ }^{26}$ Transcription of CCL2 in cells transfected with CAV1-siRNA was significantly suppressed (approx. 2-fold) in comparison with non-transfected cells and cells transfected with control siRNA (Fig. 3C). The CAV1 knockdown caused no significant change in the concentration of secreted CCL2 in comparison with cells transfected with control siRNA and non-transfected cells (Fig. 3D).

\section{Discussion}

Many chemoattractants overproduced by cells residing in the synovium enhance leukocyte migration into the joints, which is the main characteristic of RA. The activation of FLSs is believed to be the key process promoting inflammation and joint damage in RA. ${ }^{5}$ In this study, we checked whether the knockdown of $C A V 1$, a regulator of intracellular signal transduction, affects CCL2 expression in sfd-FLSs obtained from RA synovial fluid. ${ }^{14}$ Our results showed that under the study conditions, the CAV1 knockdown significantly impaired IL-1 $\beta$-induced CCL2 expression, but only at transcript level. To date, there have been no similar reports on associations between $C A V 1$ and chemoattractants in cells important for RA development.

Majkova et al. showed that the CAV1 knockdown almost completely prevented CCL2 expression induced by 3,3',4,4'-tetrachlorobiphenyl (PCB77) in endothelial cells. ${ }^{28}$ These results were also confirmed in vivo on LDL- $\mathrm{R}^{-/-} / \mathrm{Cav1}^{-/-}$mice. A similar expression pattern was observed at both the transcript and protein level for IL-6 - another important molecule stimulating immune responses. Their results suggested that $C A V 1$ can be a common regulator of inflammatory responses caused by toxic properties of PCB77. It was also presented that PCB77-induced CCL2 expression depends on p38 mitogen-activated protein kinases (MAPK) and c-Jun amino-terminal kinase (JNK). They suggested that functional caveolae are essential in order to regulate CCL2 expression. ${ }^{28}$

$\mathrm{Li}$ et al. reported that $C A V 1$ expression may enhance inflammation by an increased leukocyte influx. In their study on retinitis in $\mathrm{Cav1}^{-/-}$mice, the levels of CCL2, CXCL1, IL-6, and IL-1 $\beta$, which are downstream effectors of the Tolllike receptor (TLR)-4 signaling pathway, were reduced. ${ }^{29}$ It is highly possible that $C A V 1$ could also be responsible for the expression of pro-inflammatory molecules also in FLSs. TLR-2, -3 and -4 are predominant and the most functional TLRs in this cell type. ${ }^{29}$ An inflamed joint and its milieu are the source of different potential TLR ligands and CAV1 may be one of these proteins which efficiently enhance TLR4 signaling, followed by the production of pro-inflammatory cytokines. ${ }^{30}$ Another point of importance is that CAV1 phosphorylation on Tyr14 induces its interaction with TLR4 and leads to nuclear factor kappa-light-chain-enhancer of activated $B$ cells (NF- $\mathrm{B}$ ) activation. ${ }^{31}$
Lv et al. reported that the overexpression of CAV1 in mouse lung alveolar type-1 cells enhances the phosphorylation and expression of p38 MAPK and NF-kB, which induce the expression of inflammatory cytokines such as IL- 6 and TNF- $\alpha$. Thereby, it was additionally proven that $C A V 1$ could be an important activator of inflammation. ${ }^{32}$

Based on molecular studies on the promoter region of CCL2, some cis-regulatory elements and trans-acting factors involved in CCL2 expression were identified. It was shown that 2 NF-kB-binding sites located $2.6 \mathrm{~kb}$ from the transcription initiation site are pivotal to CCL2 expression in response to stimuli such as IL- $1 \beta$ and TNF- $\alpha .{ }^{33,34}$ This fact together with the study by Garrean et al. and studies mentioned above point to the importance of CAV1 in NF-kB activation, which could partially explain the inhibition of CCL2 expression at transcript level in sfd-FLSs with diminished CAV1 expression. ${ }^{35}$

Studies showing the pro-inflammatory character of CAV1 are not consistent with the results presented by Gardner et al. ${ }^{36}$ In a study on the hepatotoxic activity of paracetamol, they revealed an elevated expression of CCL2 in the liver obtained from $\mathrm{Cav1}^{-/-}$mice. In this case, this was linked to the "positive" effect of monocyte influx, leading to repairing processes mediated by macrophages with an anti-inflammatory phenotype. ${ }^{36}$

Looking at all the research conducted on animal models, it can be concluded that CCL2 expression is CAV1dependent, but only to a certain extent. Our results suggest that CCL2 secretion by sfd-FLSs obtained from RA patients could be regulated at the posttranslational level. It would be worth seeing if the disproportions between CCL2 transcript and protein levels could be a result of the activity of some regulators controlled by the particular CAV1 isoform. This is due to the fact that only $\alpha$ isoform has a complete sequence and undergoes phosphorylation on Tyr14, which facilitates binding both inductors and effector proteins in signaling pathways. ${ }^{37}$ Kogo et al. suggested that the ratio of the 2 isoforms may vary depending on the cell type. ${ }^{38}$ We found that CAV1 $\beta$ (lacking Nterminal 31 amino acids) is predominant in sfd-FLSs. ${ }^{39}$ Therefore, it seems that the functional diversity of both isoforms should be considered when interpreting the role of $C A V 1$ in a specific molecular context.

As some stimuli may act differently on various cell types, CCL2 expression could also vary among different cells and for different kinds of stimuli, and, as was shown in our study, the secreted CCL2 level does not always correspond with its transcript level, especially in cells with an impaired CAV1 expression. ${ }^{35}$ Here, it should be also noted that in vivo, the final level of a particular cytokine is very often an effect of complex, reciprocal synergistic and antagonistic associations between other cytokines. Additionally, understanding epigenetic mechanisms (DNA methylation and histone modifications as well as regulation by small non-coding RNAs) can be helpful in current studies on the pathogenesis of RA. A recent study by $\mathrm{Li}$ et al. presented that targeting CAV1 by restoring the 
microRNA-192 levels in FLSs led to their apoptosis and the inhibition of cell proliferation, ${ }^{40}$ and possibly by regulating the expression of other relevant genes in a direct (chemokine (C-X-C motif) ligand 2; source: miRDB-an online database (http:// www.mirdb.org/cgi-bin/search.cgi?searchType=miRNA\&se archBox=hsa-miR-192-5p\&full=1) or indirect way. To summarize, the normalization of pathological processes in RA, preferably by aiming at one pivotal factor, is highly desirable but, so far, unattainable.

\section{Conclusions}

Our results show a significant role of CAV1 in CCL2 transcription induction in RA sfd-FLSs under the influence of IL-1 $\beta$. However, processes involving $C A V 1$ still require more profound research into a broader spectrum of cytokines in auto-inflammatory disorders.

\section{References}

1. Bax M, van Heemst J, Huizinga TW, Toes RE. Genetics of rheumatoid arthritis: What have we learned? Immunogenetics. 2011;63(8): 459-466.

2. Bartok B, Firestein GS. Fibroblast-like synoviocytes: Key effector cells in rheumatoid arthritis. Immunol Rev. 2010;233(1):233-255.

3. Iwanaga T, Shikichi M, Kitamura H, Yanase H, Nozawa-Inoue K. Morphology and functional roles of synoviocytes in the joint. Arch Histol Cytol. 2000;63(1):17-31.

4. Filer A. The fibroblast as a therapeutic target in rheumatoid arthritis. Curr Opin Pharmacol. 2013;13(3):413-419.

5. Iwamoto T, Okamoto $\mathrm{H}$, Toyama $\mathrm{Y}$, Momohara $\mathrm{S}$. Molecular aspects of rheumatoid arthritis: Chemokines in the joints of patients. FEBS J. 2008;275(18):4448-4455.

6. Juarez M, Filer A, Buckley CD. Fibroblasts as therapeutic targets in rheumatoid arthritis and cancer. Swiss Med Wkly. 2012;142:w13529.

7. Bottini N, Firestein GS. Duality of fibroblast-like synoviocytes in RA: Passive responders and imprinted aggressors. Nat Rev Rheumatol. 2013;9(1):24-33.

8. Harigai M, Hara M, Yoshimura T, Leonard EJ, Inoue K, Kashiwazaki S. Monocyte chemoattractant protein-1 (MCP-1) in inflammatory joint diseases and its involvement in the cytokine network of rheumatoid synovium. Clin Immunol Immunopathol. 1993;69(1):83-91.

9. Thurlings RM, Wijbrandts CA, Bennink RJ, et al. Monocyte scintigraphy in rheumatoid arthritis: The dynamics of monocyte migration in immune-mediated inflammatory disease. PLOSONE. 2009;4(11):e7865.

10. Jorgensen C, Apparailly F. Prospects for gene therapy in inflammatory arthritis. Best Pract Res Clin Rheumatol. 2010;24(4):541-552.

11. Fujimoto $T$, Kogo $H$, Nomura R, Une T. Isoforms of caveolin-1 and caveolar structure. J Cell Sci. 2000;113(19):3509-3517.

12. Anderson RG. The caveolae membrane system. Annu Rev Biochem. 1998;67:199-225.

13. Torres VA, Tapia JC, Rodríguez DA, et al. Caveolin-1 controls cell proliferation and cell death by suppressing expression of the inhibitor of apoptosis protein survivin. J Cell Sci. 2006;119(9):1812-1823.

14. Boscher C, Nabi IR. Caveolin-1: Role in cell signaling. Adv Exp Med Biol. 2012;729:29-50.

15. Luanpitpong S, Talbott SJ, Rojanasakul Y, et al. Regulation of lung cancer cell migration and invasion by reactive oxygen species and caveolin-1. J Biol Chem. 2010;285(50):38832-38840.

16. Head BP, Insel PA. Do caveolins regulate cells by actions outside of caveolae? Trends Cell Biol. 2006;17(2):51-57.

17. Kimura A, Mora S, Shigematsu S, Pessin JE, Saltiel AR. The insulin receptor catalyzes the tyrosine phosphorylation of caveolin-1.J Biol Chem. 2002;277(33):30153-30158.

18. Wang XM, Kim HP, Nakahira K, Ryter SW, Choi AM. The heme oxygenase-1/carbon monoxide pathway suppresses TLR4 signaling by regulating the interaction of TLR4 with caveolin-1. JImmunol. 2009;182(6): 3809-3818.
19. Catalán V, Gómez-Ambrosi J, Rodríguez A, et al. Expression of caveolin-1 in human adipose tissue is upregulated in obesity and obesity-associated type 2 diabetes mellitus and related to inflammation. Clin Endocrinol (Oxf). 2008;68(2):213-219.

20. Zhang PX, Murray TS, Villella VR, et al. Reduced caveolin-1 promotes hyperinflammation due to abnormal heme oxygenase-1 localization in lipopolysaccharide-challenged macrophages with dysfunctional cystic fibrosis transmembrane conductance regulator. J Immunol. 2013;190(10):5196-5206.

21. Takizawa N, Sawada S, Chosa N, Ishisaki A, Naruishi K. Secreted caveolin-1 enhances periodontal inflammation by targeting gingival fibroblasts. Biomed Res. 2013;34(1):1-11.

22. Arnett FC, Edworthy SM, Bloch DA, et al. The American Rheumatism Association 1987 revised criteria for the classification of rheumatoid arthritis. Arthritis Rheum. 1988;31(3):315-324.

23. Aletaha D, Neogi T, Silman AJ, et al. 2010 Rheumatoid arthritis classification criteria: an American College of Rheumatology/European League Against Rheumatism collaborative initiative. Arthritis Rheum. 2010;62(9):2569-2581.

24. Stebulis JA, Rossetti RG, Atez FJ, Zurier RB. Fibroblast-like synovial cells derived from synovial fluid. J Rheumatol. 2005;32(2):301-306.

25. Scanu A, Oliviero F, Braghetto $L$, et al. Synoviocyte cultures from synovial fluid. Reumatismo. 2007;59(1):66-70.

26. Baugé C, Girard N, Leclercq S, Galéra P, Boumédiene K. Regulatory mechanism of transforming growth factor beta receptor type Il degradation by interleukin-1 in primary chondrocytes. Biochim Biophys Acta. 2012;1823(5):983-986.

27. Hayer A, Stoeber M, Ritz D, Engel S, Meyer HH, Helenius A. Caveolin-1 is ubiquitinated and targeted to intralumenal vesicles in endolysosomes for degradation. J Cell Biol. 2010;191(3):615-629.

28. Majkova Z, Smart E, Toborek M, Hennig B. Up-regulation of endothelial monocyte chemoattractant protein-1 by coplanar PCB77 is caveolin-1-dependent. Toxicol Appl Pharmacol. 2009;237(1):1-7.

29. Li X, Gu X, Boyce TM, Zheng M, et al. Caveolin-1 increases proinflammatory chemoattractants and blood-retinal barrier breakdown but decreases leukocyte recruitment in inflammation. Invest Ophthalmol Vis Sci. 2014;55(10):6224-6234.

30. Mirza MK, Yuan J, Gao XP, et al. Caveolin-1 deficiency dampens Tolllike receptor 4 signaling through eNOS activation. Am J Pathol. 2010; 176(5):2344-2351.

31. Jiao H, Zhang Y, Yan Z, et al. Caveolin-1 Tyr14 phosphorylation induces interaction with TLR4 in endothelial cells and mediates MyD88dependent signaling and sepsis-induced lung inflammation. J Immunol. 2013;191(12):6191-6199.

32. Lv XJ, Li YY, Zhang YJ, Mao M, Qian GS. Over-expression of caveolin-1 aggravate LPS-induced inflammatory response in AT-1 cells via upregulation of CPLA2/p38 MAPK. Inflamm Res. 2010;59(7):531-541.

33. Yadav A, Saini V, Arora S. MCP-1: Chemoattractant with a role beyond immunity: A review. Clin Chim Acta. 2010;411(21-22):1570-1579.

34. Ueda A, Okuda K, Ohno S, et al. NF-kappa B and Sp1 regulate transcription of the human monocyte chemoattractant protein-1 gene. J Immunol. 1994;153(5):2052-2063.

35. Garrean S, Gao XP, Brovkovych V, et al. Caveolin-1 regulates NF-kappaB activation and lung inflammatory response to sepsis induced by lipopolysaccharide. J Immunol. 2006;177(7):4853-4860.

36. Gardner CR, Gray JP, Joseph LB, et al. Potential role of caveolin-1 in acetaminophen-induced hepatotoxicity. Toxicol Appl Pharmacol. 2010;245(1):36-46.

37. Li S, Seitz R, Lisanti MP. Phosphorylation of caveolin by src tyrosine kinases. The alpha-isoform of caveolin is selectively phosphorylated by v-Src in vivo. J Biol Chem. 1996;271(7):3863-3868.

38. Kogo H, Aiba T, Fujimoto T. Cell type-specific occurrence of caveolin-1alpha and -1beta in the lung caused by expression of distinct mRNAs. J Biol Chem. 2004;279:25574-25578.

39. Scherer PE, Tang Z, Chun M, Sargiacomo M, Lodish HF, Lisanti MP. Caveolin isoforms differ in their $\mathrm{N}$-terminal protein sequence and subcellular distribution: Identification and epitope mapping of an isoform-specific monoclonal antibody probe. J Biol Chem. 1995; 270(27):16395-16401.

40. Li S, Jin Z, Lu X. MicroRNA-192 suppresses cell proliferation and induces apoptosis in human rheumatoid arthritis fibroblast-like synoviocytes by downregulating caveolin 1. Mol Cell Biochem. 2017;432(1-2): 123-130. 